\title{
Photon confinement in one-dimensional photonic quantum-well structures of nanoporous silicon
}

\author{
S.H. Xu, Z.H. Xiong, L.L. Gu, Y. Liu, X.M. Ding, J. Zi, X.Y. Hou* \\ Surface Physics Laboratory (National Key Lab), Fudan University, Shanghai 200433, People's Republic of China
}

Received 6 November 2002; accepted 17 December 2002 by K.-A. Chao

\begin{abstract}
Various combinations of periodically assembled nanoporous silicon layers with different refractive indices and thicknesses have been used to fabricate one-dimensional photonic quantum well structures, in which both the well and barrier regions consist of photonic crystals. The structures are operational in the regime of visible light. Quantized states resulting from the photonic confinement effect are observed, consistent with calculations using the transfer-matrix method. Behaviors of the photons in the structures can be well described by the effective wave-vector approach.
\end{abstract}

(C) 2003 Elsevier Science Ltd. All rights reserved.

PACS: 42.70.Qs; 68.65. + g; 78.66. $-\mathrm{w}$

Keywords: A. Nanostructures; D. Optical Properties; E. Light absorption and reflection

It has been well established that, in analogy to the electrons in a quantum well $(\mathrm{QW})$ or superlattice $[1,2]$, the photons in a proper assembly of photonic crystals (PCs) with different band gaps are confined to quantized energy states, or referred to as being in a photonic quantum well structure (PQWS) [3-10]. Early explorations in the millimeter-wave region gave rise to the emergence of two- and threedimensional PQWSs $[3,4,9]$. The transmissivity of a confined photonic state can be as high as unity, just like resonant tunneling of an electron through a semiconductor QW system. Obviously, the novel features of PQWSs are attractive for both fundamental and application studies.

The simplest way to form a one-dimensional (1D) PQWS is to use a homogenous dielectric slab as the photonic well, sandwiched between two PCs as the photonic barriers [10]. But it is difficult to observe the photonic band gap (PBG) or QW effect in such a 1D PQWS and the structure arouses little interest actually [8]. Alternatively, a properly designed PC can be used as the well in a PQWS [7]. In that case, one may expect the QW effect observable and the number of the confined photonic states tunable by

\footnotetext{
* Corresponding author. Tel.: +86-2165642131; fax: + 86 2164191419.

E-mail address: xyhou@fudan.edu.cn (X.Y. Hou).
}

adjusting the number of the PC periods. This may lead to applications of the new structure in high-performance optoelectronic devices such as high-frequency PBG devices, multi-channel filters, etc. Further experimental investigation on the latter 1D PQWS is thus needed.

In this communication, we report on realization of the structure by using porous silicon as the constituent material for all the PCs. The 1D PQWSs fabricated are operational in the regime of visible light and can be well described by the effective wave-vector approach [11,12] initially developed for description of the phonon confinement effect in the electronic QWs. The theoretically predicted [7] nonequivalence of the number of the confined photonic states with the photonic well periods in case that the photonic barrier does not completely confine a photonic band of the photonic well has also been experimentally confirmed.

Exactly speaking, the porous silicon constituting the PCs studied in the present experiment is nanoporous silicon $(\mathrm{nPSi})^{1}$, prepared by means of pulsed electrochemical etching [13]. Heavily doped $(\sim 0.01 \Omega \mathrm{cm})$ p-type $\mathrm{Si}(001)$

\footnotetext{
${ }^{1}$ Porous silicon is usually divided into three categories by the sizes of the silicon pores: nanoporous $(<2 \mathrm{~nm})$, mesoporous $(10 \sim 100 \mathrm{~nm})$ and macroporous $(0.1 \sim 20 \mu \mathrm{m})$. In our experiment, nanoporous silicon is the main component.
} 
wafers were used as the substrates, and the etchant was a $\mathrm{HF}(48 \%)-\mathrm{C}_{2} \mathrm{H}_{5} \mathrm{OH}(99 \%)-\mathrm{H}_{2} \mathrm{O}$ solution $(1: 1: 2$ by volume). As was described in detail elsewhere [14], both thickness $L$ and refractive index $n$ of a nPSi layer could be well controlled by changing the duration time and current density for pulsed etching. Proper assemblies of different nPSi layers A, B, C and D could result in the formation of two PQWSs, $(\mathrm{AB})_{n} /(\mathrm{CD})_{m} /(\mathrm{AB})_{n}(\mathrm{QW} 1)$ or $(\mathrm{CD})_{m} /(\mathrm{AB})_{n} /$ $(\mathrm{CD})_{m}(\mathrm{QW} 2)$, as shown in the inset of Fig. 1. Here $m$ and $n$ were the periods of the nPSi layers to form the PCs, both set to 5 in the present case. And the refractive indices and thicknesses of the nPSi layers were $n_{\mathrm{A}}=2.2$, $L_{\mathrm{A}}=0.07 \mu \mathrm{m} ; \quad n_{\mathrm{B}}=1.4, \quad L_{\mathrm{B}}=0.09 \mu \mathrm{m} ; \quad n_{\mathrm{C}}=2.2$, $L_{\mathrm{C}}=0.085 \mu \mathrm{m}$; and $n_{\mathrm{D}}=1.4, L_{\mathrm{D}}=0.13 \mu \mathrm{m}$, respectively. The validity of the data derived was further checked by scanning electron microscopic measurements [15] and selfconsistent fitting of the measured reflectance spectra based on the transfer matrix method (TMM) [16,17].

The reflectance spectra were measured by using a $100 \mathrm{~W}$ tungsten iodide lamp as the light source, incident at about $8^{\circ}$ off normal. The reflected light was normally detected by a spectrophotometer consisting of a $0.275 \mathrm{~m}$ double monochromator, a photomultiplier tube and a lock-in amplifier.

The photonic band structures of the AB and CD PCs are shown in Fig. 1. One can see that the PBGs of the AB and $\mathrm{CD}$ PCs lie in the photon energy regions of $1.445 \sim 1.923 \mathrm{eV}$ and $1.935 \sim 2.567 \mathrm{eV}$, respectively, overlapping at around $1.93 \mathrm{eV}$. As one will see below, for a PQWS consisting of the two sorts of PCs, sharp confined modes are observable in the energy region where $\mathrm{AB}$ and $\mathrm{CD}$ act as the photonic barrier and well, respectively. Outside this region, there exists dimmed optical oscillation instead.

Figs. 2 and 3 show the calculated and measured reflectance spectra, respectively, where panels I, II, III and IV correspond to the $\mathrm{AB}$ PC, CD PC, QW1 and $\mathrm{QW} 2$, respectively. One can see that the experimental spectra taken from the two PCs are well consistent with the theoretical ones: the $\mathrm{AB} \mathrm{PC}$ is highly reflective from 500 to $650 \mathrm{~nm}(1.911 \sim 2.485 \mathrm{eV})$; while for $\mathrm{CD}$, the high-reflectance region appears at above $650 \mathrm{~nm}$. They correspond to the PBGs of the two PCs, respectively. It can also be seen that the PBG of QW1 is actually the sum of the PBGs of $\mathrm{AB}$ and $\mathrm{CD}$, covering the region from 500 to $850 \mathrm{~nm}(1.462 \sim 2.485 \mathrm{eV})$, as predicted in Ref. [6]. Within this region, there emerge three narrow peaks, located at $508 \mathrm{~nm}(2.446 \mathrm{eV}), 544 \mathrm{~nm}(2.284 \mathrm{eV})$ and $588 \mathrm{~nm}(2.113 \mathrm{eV})$, respectively, all in the PBG region of the $\mathrm{AB}$ PC. But the peak intensities experimentally measured are not as high as theoretically calculated. This can be ascribed to the influence of the nPSi material itself, as will be further discussed later. Outside the PBG of $\mathrm{AB}$, there are two broad peaks, located at $656 \mathrm{~nm}(1.894 \mathrm{eV})$ and $766 \mathrm{~nm}(1.622 \mathrm{eV})$, respectively.

To explain the results obtained, distribution of the

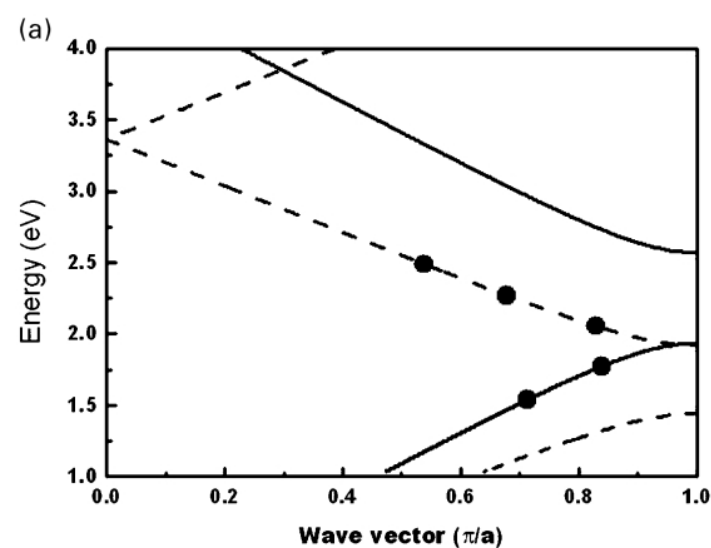

(b)

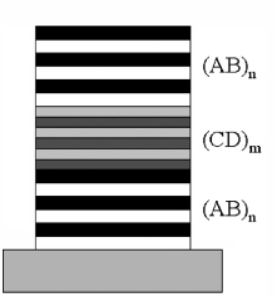

Fig. 1. (a) Photonic band structures of $\mathrm{AB}$ (dashed line) and $\mathrm{CD}$ (solid line) PCs. The black dots on the lines are the confined states experimentally measured from QW1 and QW2. Refer to the text for details. (b) Schematic of a one-dimensional PQWS.

electric field in QW1 is calculated by TMM, as shown in Fig. 4. The varied refractive indices in the structure is shown in the top panel, where subscripts $\mathrm{A}, \mathrm{H}(\mathrm{L})$ and $\mathrm{S}$ represent air, high (low) refractive index layer and silicon bulk, respectively. The magnitude of the incident electric field is set to be unity. The wavelengths of the incident light are 499, 547, 605, 701 and $832 \mathrm{~nm}$ for panels $\mathrm{I} \sim \mathrm{V}$,

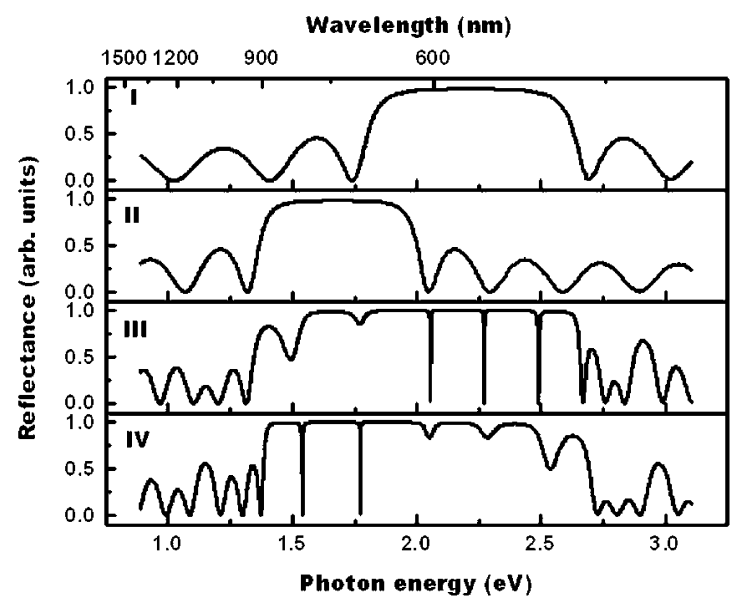

Fig. 2. Reflectance spectra calculated using TMM for (I) AB PC, (II) $\mathrm{CD} \mathrm{PC}$, (III) $(\mathrm{AB})_{5} /(\mathrm{CD})_{5} /(\mathrm{AB})_{5}$ and $(\mathrm{IV})(\mathrm{CD})_{5} /(\mathrm{AB})_{5} /(\mathrm{CD})_{5}$. Here letters $\mathrm{A}, \mathrm{B}, \mathrm{C}$ and $\mathrm{D}$ represent different dielectric slabs, and subscript 5 means each PC consists of 5 periods. 


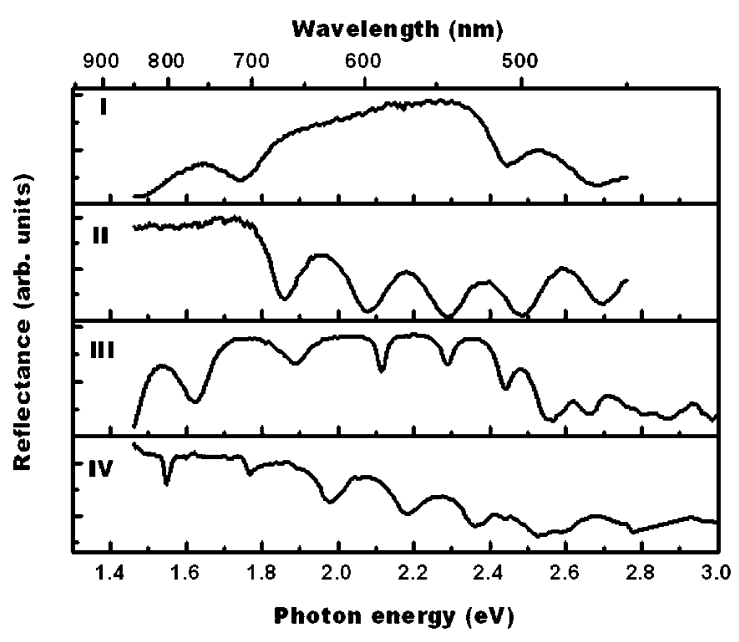

Fig. 3. Reflectance spectra experimentally measured from the same structures as in Fig. 2.

respectively, corresponding to either narrow or broad peaks in the calculated reflectance spectra. One can see that the electric fields are mainly confined in the CD PC though there exist attenuated wings penetrating into the adjacent $A B$ layers for the shorter wavelengths 499, 547 and $605 \mathrm{~nm}$. Evidently, in the PBG region of the AB PC $(1.3 \sim 1.8 \mathrm{eV})$, the electromagnetic (EM) waves can transit not the $\mathrm{AB}$ but CD PC only. That is to say, the AB PCs are two barriers and $\mathrm{CD}$ a well, which leads to confining of the photons to quantized energies, like electrons and phonons in semiconductor QW structures. The defect-like states in the PBG region of the $\mathrm{AB} \mathrm{PC}$ are due to confinement of the photons in the CD layer. As shown in the reflectance spectra (Fig. 2), the transmissivity for a confined state can be as high as

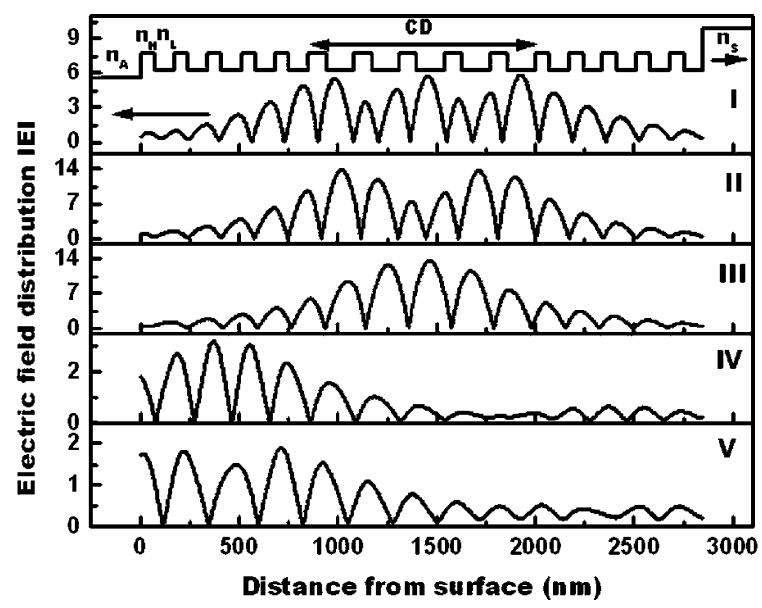

Fig. 4. Distribution of the electric field in QW1 $\left((\mathrm{AB})_{5} /(\mathrm{CD})_{5} /\right.$ $\left.(A B)_{5}\right)$. The varied refractive indices in the structure are shown in the top panel. Wavelengths of the incident light used for the 5 panels are (from top to bottom) 499, 547, 605, 701 and $832 \mathrm{~nm}$, respectively. $n_{\mathrm{A}}, n_{\mathrm{H}}\left(n_{\mathrm{L}}\right)$ and $n_{\mathrm{S}}$ are the refractive indices of air, high (low) refractive index layer and silicon bulk, respectively. unity. That hints the EM wave can pass through the PQWS in a way similar to resonant tunneling of the electrons in a semiconductor QW structure [8]. One may also find that different incident lights can result in different electric field distributions, namely, three, two and one envelope oscillation peaks for the incident lights of 499, 547 and $605 \mathrm{~nm}$, respectively. This indicates that the different confined photonic states are associated with the different electric field oscillation modes. The deep states (those in the middle of PBG) are much more confined than the shallow ones (those near the band edges). It is evident in the reflectance spectra of the PQWS that the more confined the electric field, the narrower the peak width. The electric fields are confined in the top AB PC for the incident lights of $701 \mathrm{~nm}$ $(1.772 \mathrm{eV})$ and $832 \mathrm{~nm}(1.493 \mathrm{eV})$. This is because the two incident lights lie in the PBG region of the CD PC so that the EM waves can travel in the top $\mathrm{AB}$ PC but be reflected by the CD PC. If the incident wave cannot be completely confined by the photonic barrier and oscillation of the incident and reflected waves occurs in the top AB PC, then the broad and low-transmission peaks will appear. The different broad peaks are associated with the different oscillation modes, as is discussed for confined photonic states. The number of the confined states is not equal to the number of the photonic well periods, as Qiao et al. predicted in Ref. 7. This is because the photonic band of the CD PC is not entirely inside the PBG of the AB PC so the barriers do not confine the five states completely. Two of them run out of the photonic quantum well and submerge in the low wavelength region of the PBG of the well.

For QW2, the CD PCs are the barriers and AB is the well in the energy region from 1.93 to $2.57 \mathrm{eV}$. Do confined photonic states exist in this energy region, similar to what discussed above for QW1? The calculated and measured results (Figs. 2(IV) and 3(IV)) have proven that is the case. Two sharp peaks, corresponding to the confined photonic states at the wavelengths of $702 \mathrm{~nm}(1.770 \mathrm{eV})$ and $802 \mathrm{~nm}$ $(1.549 \mathrm{eV})$, respectively, appear in the PBG region of the CD PC. In other words, the electric field is confined in the AB PC, the photonic well of QW2. Thus, it seems clear that confined photonic states would be present if one sets the PBG regions of the well and barrier PCs so that EM waves can travel not in the barrier PCs but in the well PC only. The conclusion is likely applicable to not only visible light, but also other interested wavelength regions.

A confined photonic state can be regarded as a standing wave, describable by an effective wave-vector $q_{\mathrm{eff}}=i 2 \pi / L$ $[11,12]$. Here $i$ is the index of the confined mode and $L$ the width of the well. Since the electric field may penetrate into the adjacent barrier layers to some extent (see Fig. 4), $L$ would be somewhat larger than the nominal width of the well. It can be expressed as $L=n a+\delta$, where $a$ is the thickness of each well layer, $n$ the number of the layers, and $\delta$ the penetration depth of the electric field into the adjacent barrier layers. The effective wave-vector for a confined 
mode is thus given by

$q_{\mathrm{eff}}=i \frac{2 \pi}{n a+\delta}$

A set of $\delta$ 's, 200, 260 and $320 \mathrm{~nm}$, can then be derived from the above equation for the confined photonic states of 499 , 547 and $605 \mathrm{~nm}$, respectively, to fit the bulk dispersion of the photonic well shown in Fig. 1. The penetration parameters so obtained for the different confined photonic states are qualitatively consistent with the distributions of the electric fields in that the deep confined photonic states are much more confined than the shallow ones. Application of the above equation to QW2 can lead to the same conclusion. The good agreement of the experimental results with the theoretical ones indicates that effective wavevector, a concept for description of the phonons in the electronic QW structures, is applicable to the photonic QW structures as well.

It should be mentioned that the measured results deviate from the calculated ones to some extent in the following aspects. First, the transmissivity of the confined states is not as high as approaching unity. Second, some of the confined states emerge as broad peaks rather than narrow spikes. And third, the two broad peaks shift to lower wavelengths. These discrepancies can be attributed to the factors not taken into account in the calculations, including absorption of the EM waves by the nPSi layers, rough interfaces between the nPSi layers, and inhomogeneity in nPSi layer thickness $[18,19]$. It is therefore understandable that the peaks experimentally observed cannot be as sharp as those calculated.

In summary, nPSi-based, 1D PQWSs have been fabricated by sandwiching a photonic well PC between two photonic barrier PCs. The experimentally measured reflectance spectra are consistent with those calculated by TMM. Quantized states resulting from the photonic confinement effect are observed in the PBG of the barrier PCs. It is found that the more confined the electric field, the narrower is the peak width. The effective wave-vector approach is applicable to description of the behaviors of the photons in the PQWSs.

This work was supported by the National Natural Science Foundation of China (Grant No. 59832100) and CNKBRSF (Grant No. 2001CB610408).

\section{References}

[1] L. Esaki, R. Tus, IBM J. Res. Dev. 14 (1970) 61.

[2] E.E. Mendez, K. von Klitzing (Eds.), Physics and Application of Quantum Wells and Superlattices, Plenum, New York, 1987.

[3] S.Y. Lin, G. Arjavalingam, J. Opt. Soc. Am. B 11 (1994) 2124

[4] S.Y. Lin, G. Arjavalingam, Opt. Lett. 18 (1993) 1666.

[5] S.-Y. Lin, V.M. Hietala, S.K. Lyo, A. Zaslavsky, Appl. Phys. Lett. 68 (1996) 3233.

[6] J. Zi, J. Wan, C. Zhang, Appl. Phys. Lett. 73 (1998) 2084.

[7] F. Qiao, C. Zhang, J. Wang, J. Zi, Appl. Phys. Lett. 77 (2000) 3698.

[8] Y. Jiang, C. Niu, D.L. Liu, Phys. Rev. B 59 (1999) 9981.

[9] S. Yano, Y. Segawa, J.S. Bae, K. Mizuno, H. Miyazaki, K. Ohtaka, S. Yamaguchi, Phys. Rev. B 63 (2001) 153316.

[10] H. Miyazaki, Y. Jimba, C.-Y. Kim, T. Watanabe, J. Phys. Soc. Jpn 65 (1996) 3842.

[11] J. Zi, K. Zhang, X. Xie, Prog. Surf. Sci. 54 (1997) 69.

[12] B. Jusserand, M. Cardona, in: M. Cardona, G. Guntherodt (Eds.), Light Scattering in Solids V, Springer, New York, 1989.

[13] X.Y. Hou, H.L. Fan, L. Xu, F.L. Zhang, M.Q. Li, M.R. Yu, X Wang, Appl. Phys. Lett. 68 (1996) 2323.

[14] S.H. Xu, Z.H. Xiong, L.L. Gu, Y. Liu, X.M. Ding, J. Zi, X.Y. Hou, Appl. Phys. A, in Press.

[15] Z.H. Xiong, L.S. Liao, X.M. Ding, S.H. Xu, Y. Liu, L.L. Gu, F.G. Tao, S.T. Lee, X.Y. Hou, Appl. Phys. A 74 (2002) 807.

[16] P. Yeh, A. Yariv, C.S. Hong, J. Opt. Soc. Am. B 67 (1976) 423.

[17] Z. Knittl, Optics of Thin Films, Wiley, London, 1976.

[18] S. Setzu, P. Ferrand, R. Romestain, Mat. Sci. Engng. B 69-70 (2000) 34

[19] G. Vincent, Appl. Phys. Lett. 64 (1994) 2367. 